\title{
8.6 LOW-FREQUENCY CUTOFFS IN SYNCHROTRON SPECTRA AND THE OPTICAL SPECTRUM OF NP 0532*
}

\author{
STEPHEN L. O'DELL and L. SARTORI \\ Department of Physics and Center for Space Research, \\ Massachusetts Institute of Technology, Cambridge, Mass., U.S.A.
}

\begin{abstract}
We point out the existence of a natural low-frequency cutoff in the spectrum of a synchrotron source. The turnover frequency is $e B \mid m c\langle\sin \psi\rangle$, where $\psi$ is the pitch angle for the electron motion. The spectrum below the turnover goes as $v^{n}$, where $n$ is the index in the electron energy distribution. For the Crab pulsar, a model with $B \sim 10^{6}-10^{7} \mathrm{G}$ and $\langle\sin \psi\rangle \sim 10^{-3}-10^{-2}$ provides a plausible explanation for the turnover observed in the infrared.
\end{abstract}

It is commonly believed that without self-absorption, the low-frequency spectrum of a non-thermal source can be no steeper than $v^{1 / 3}$. This assertion is based on the fact that the spectrum of a single particle behaves as $v^{1 / 3}$ at low frequencies. However, that behaviour does not extend to zero frequency, but cuts off abruptly at the fundamental frequency

$$
v_{0}=v_{B} / \gamma \xi^{2}
$$

where $v_{B}$ is the cyclotron frequency and $\xi$ is the sine of the pitch angle. (Actually the spectrum is discrete at the low-frequency end but with a distribution in energy this will never be noticed.)

If one takes account of this cut-off, and folds in the usual power law distribution in electron energy, $\mathrm{d} N / \mathrm{d} \gamma \sim \gamma^{-n}$, one finds that the low-frequency spectrum in fact has the form

$$
I_{v} \sim v^{n} \sim v^{2 \alpha+1}
$$

where $\alpha$ is the index that characterizes the high-frequency spectrum. The turnover frequency is approximately

$$
v_{t} \sim v_{B} / \xi
$$

where $\xi$ is an average pitch angle for the emitting particles. For quasi-isotropic distributions in pitch angle, $v_{t}$ is just the cyclotron frequency, but if small pitch angles are predominant, the turnover is shifted to higher frequency.

Notice further that below the turnover, radiation of frequency $v$ comes only from electrons whose fundamental frequency is lower than $v$; these must have $\gamma>\gamma_{\min }$, where

$$
\gamma_{\min }(v, \xi)=v_{B} / v \xi^{2} .
$$

That is to say, the low-frequency end of the spectrum comes only from electrons of

* Research supported in part by NASA and also in part by the National Science Foundation. 
high energy. Low-energy electrons (if they are present) contribute only in the neighborhood of $v_{t}$. (For small pitch angles, 'low' energy means $\gamma \lesssim \xi^{-1}$; any such electron is nonrelativistic in the frame in which its motion is circular.)

This effect, which we call cyclotron turnover, must occur in every synchrotron source. (If the electron energy distribution should be truncated at the low end, this merely flattens the spectrum near the turnover.) However, cyclotron turnover must compete with synchrotron self-absorption in cutting off the spectrum. Clearly, the effect which sets in at higher frequency will be the one observed. If the angular size of the source and the typical pitch angles are known, one can say unequivocally which effect dominates. The condition is

$$
\begin{array}{rll}
C(\alpha) v_{m}^{4} S\left(v_{m}\right)^{-2} \theta^{4} \xi^{-2} & <1 & \text { self-absorption } \\
& >1 & \text { cyclotron turnover }
\end{array}
$$

where $v_{m}$ is the observed turnover frequency, $S\left(v_{m}\right)$ is the observed flux at the turnover frequency, and $C(\alpha)$ is a slowly varying function of the spectral index. Briefly, what this says is that we calculate $B_{\perp}$ assuming self-absorption, and use that field to find $v_{t}$ using equation (3). If the $v_{t}$ so determined is higher than $v_{m}$, the assumption of selfabsorption is inconsistent and only cyclotron turnover is a possible explanation. (It must be verified in any case that the field implied by the favored mechanism is reasonable for the physical object involved.)

Remark: the standard formulae for synchrotron self-absorption have been used. It is not obvious that these are valid in the case of very small pitch angles; we are looking into this point.

We are considering the applicability of cyclotron turnover to a number of objects. I shall discuss here only the Crab pulsar, for which $v_{m} \approx 8 \times 10^{14} \mathrm{~Hz}$ (Oke, 1969; Neugebauer et al., 1969.) The precise location of the turnover is quite uncertain because of large and uncertain reddening corrections. The angular size is not known, but one can set a reasonable upper limit by assuming that the source region is roughly within the light cylinder, and subtends at the center of the neutron star an angle no greater than $2 \pi \delta$, where $\delta$ is the observed duty cycle. This makes $\theta \lesssim 10^{-14} \mathrm{rad} \approx$ $\approx 2 \times 10^{-9} \mathrm{sec}$ of arc.

For emission close to the star, criterion (5) favors selfabsorption. However, the field so determined is much too small and such a model is not tenable. For emission near the light cylinder, cyclotron turnover is favored for all $\xi \lesssim 0.1$. This value is about the most the pitch angles could be; otherwise the duty cycle of the pulsar could not be as small as observed. Several arguments suggest that the relevant values of $\xi$ should be considerably smaller than 0.1 . Among them are:

acceleration preferably along field lines adiabatic invariance radiative losses deplete the supply of high- $\xi$ electrons.

It therefore seems to us that cyclotron turnover is the more likely explanation for the turnover in the pulsar spectrum, although selfabsorption is not strictly excluded. 
For the pulsar, Equation (3) gives

$$
B / \bar{\xi} \approx 3 \times 10^{8} \mathrm{G}
$$

so with $\bar{\xi}$ between $10^{-2}$ and $10^{-3}$ one gets fields between $3 \times 10^{5}$ and $3 \times 10^{6} \mathrm{G}$, which are reasonable values. We can obtain another relation between $B$ and $\xi$ and thereby fix both of them uniquely if we are willing to make the following additional speculation. The X-ray spectrum of the pulsar appears to bend down at $v \sim 10^{18-19} \mathrm{~Hz}$. The data are not certain, but the optical and soft X-ray spectra are flatter than those of the nebula, and cannot continue very far with the same index before they would cross the nebular spectrum. Assume that this break is due to a lifetime effect in the pulsar, i.e., that $\tau_{\mathrm{rad}}\left(v_{e}\right) \sim d / c$, where $v_{e}$ is the break frequency and $d$ measures the extent of the emission region. One then obtains the following relation:

$$
B \bar{\xi} \approx 10^{15} d^{-2 / 3} v_{e}^{-1 / 3} .
$$

Putting $v_{e}=10^{18} \mathrm{~Hz}$ and $d=$ radius of light cylinder $=2 \times 10^{8} \mathrm{~cm}$ gives

$$
\begin{aligned}
& B=10^{6} \mathrm{G} \\
& \bar{\xi}=3 \times 10^{-3} .
\end{aligned}
$$

These numbers are quite encouraging. Notice they come purely from analysis of the spectrum, with no independent assumptions about the field. I should also remark that these results depend very weakly on the parameters involved; $B$ in fact varies as $v_{t}^{1 / 2} v_{e}^{-1 / 6} d^{-1 / 3}$. One cannot carry out a similar analysis if one assumes the turnover is due to self-absorption, since both $v_{t}$ and $v_{e}$ then involve the same combination $B \xi$. One can only check the two relations for consistency, but because of the fourth-power dependence of $B$ on $\theta$ such a check is not very sensitive. The values of $B$ and $\xi$ given by Equation (8) are in fact almost the same as those deduced by Shklovsky (1970) who interpreted the turnover as self-absorption. Shklovsky obtained the value of $B$ by assuming the canonical value at the surface, and a dipole dependence on $r$. However, according to Equation (5), selfabsorption is inconsistent for those values of the parameters. The electrons radiating in the vicinity of the maximum are non-relativistic in the frame that moves with the gyrocenter, and therefore the standard synchrotron theory is inapplicable.

\section{References}

Neugebauer, G., Becklin, E. E., Kristian, J., Leighton, R. B., Snellen, G., and Westphal, J. A.: 1969 Astrophys. J. Letters 156, L115.

Oke, J. B.: 1969, Astrophys. J. Letters 156, L49.

Shklovsky, I. S.: 1970, Astrophys. J. Letters 159, L77. 